\title{
Ploughing friction and nanohardness dependent on the tip tilt in nano-scratch test for single crystal gold
}

\author{
Xiaoming Liu ${ }^{\mathrm{a}, *}$, Zhanli Liu ${ }^{\mathrm{b}}$, Yueguang Wei ${ }^{\mathrm{a}}$ \\ ${ }^{a}$ LNM, Institute of Mechanics, Chinese Academy of Sciences, 100190 Beijing, China \\ ${ }^{\mathrm{b}}$ AML, School of Aerospace, Tsinghua University, 100084 Beijing, China
}

\section{A R T I C L E I N F O}

\section{Article history:}

Received 18 January 2015

Received in revised form 22 July 2015

Accepted 28 July 2015

Available online 19 August 2015

\section{Keywords:}

Nano-friction

Tilt effect

Pile-up

Local plasticity

\begin{abstract}
A B S T R A C T
Different from macro-scale scratch, in nano-scale scratch test, the scratching tip is easily tilted, which may display a considerable change of the friction behavior. The ploughing friction and nanohardness dependent on the tip tilt in nano-scratch test for single crystal gold is studied in this paper by using molecular dynamics simulations. The results show that tilting forward (backward) to the scratch direction has a larger effect on the friction coefficient than the case of tilting laterally to the scratch direction. Scratch hardness is also sensitive to the tip tilting forward (backward), while it is insensitive to the tip tilting laterally. Moreover, the local plastic behaviors near tip (pile-up, plastic area, and dislocation evolution) are also dependent on the tip tilt, which may be a major contribution to the mechanism of the nano-scale wear.
\end{abstract}

(c) 2015 Elsevier B.V. All rights reserved.

\section{Introduction}

In the nano-scratch test, both contact force and contact area are very sensitive to the local contact conditions such as the nanoscale roughness [1,2], which leads to the difficulty in the measurement with high resolution. As a result, in the nanoscale friction, the study of the relative position between the tip and the sample is of great importance. During nano-scratch process, the tip is not strictly perpendicular to the scratch surface due to the following three main factors: beam bending of the Atomic Force Microscope (AFM), substrate with a tilt angle, as well as roughness of the sample surface. Experiment [3] showed that tilt angle of the tip has significant effects on the size and shape of wear debris during nano-scratching tests. The tip tilt effects on indentation $[4,5]$ and scratch tests [6] have been studied by using finite element method, and the results show that the tip tilt leads to the change of the indentation load, contact area and contact stiffness. The change of contact area caused by the tilt is not included in Sneddons equation, commonly used in nano-indentation analysis.

Studies from continuum mechanics show, a tilt of $5^{\circ}$ can cause approximately $12 \%$ error and $30 \%$ error in measurement of hardness [5] and friction coefficient [6], respectively. These studies

\footnotetext{
* Corresponding author.

E-mail addresses: Xiaomingliu@Inm.imech.ac.cn (X. Liu), liuzhanli@mail. tsinghua.edu.cn (Z. Liu), ywei@lnm.imech.ac.cn (Y. Wei).
}

mostly attribute the influence to the difference of macro-scale yielding pattern, which is not a proper concept for nano-scale contact. In nano-scale, pile-up of material plays a critical role, different from the case in macro-scale. In order to study ploughing, Szlufarska et al. $[7,8]$ proposed an analytical model taking account of ploughing friction for single-asperity contacts, and they considered the effects of both elastic recovery and atomic pileup, but without taking account of the tip tilt effect on friction coefficient. Different from macro-scale friction, friction behavior of nano-scratch is sensitive to the detailed contact geometry: Gao et al. [9] investigated the effect of asperity shapes on the wear of nickel thin film by using molecular dynamics (MD) simulations, their results reveal that the sharp surface asperities cause more friction than that of the blunt asperities. Zhu et al. [10] gave different results, by studying the effect of indenter shape on the nanometric scratching process of copper, they showed the blunt asperities may result in more friction if the asperities are rather small. Besides, the nano-scale yielding has extra high strength, Kiely and Houston [11] showed the yield strength of gold is as high as $\sigma_{p}=7.8 \mathrm{GPa}$, which is much larger than the value of yielding at macro-scale [4-6].

Thus, it remains unknown that how the nano-friction behavior is related to the local contact condition by effects of atomic pileup, dislocation movement, and contact geometry, while these factors can be significantly changed by the tilt of the tip. Also, the tip tilt effect on scratch hardness at nano-scale has various mechanisms. These effects will be studied in this work. 


\section{Methodology}

MD simulations are carried out to study the scratch test of a conical tip over the single crystal Au (see in Fig. 1 for section views). In the present study, the package LAMMPS [12] is used for all the simulations. Tip consists of carbon atoms with diamond cubic structure, it has a much larger rigidity compared with the tested samples, thus is considered as not deformable. The tip has a cone shape with a semi-cone angle $\theta=40.0^{\circ}$. At the contact point of a real AFM tip, it always has a hemisphere with the radius in the range of nano-meters as a result of fabrication. Correspondingly, in our model, the tip curvature radius is taken as $R=10 \mathrm{~nm}$. In order to study the effect of tilt angle, tips with different tilt angles $\left(\eta_{x}\right.$ or $\eta_{z}$ ) are selected during nano-scratch process.

During indentation, periodic boundary conditions are applied in the $x$ and $z$ directions, while the bottom atoms (in the $y$ direction) are fixed, the top atoms are in a free surface (see in Fig. 1). The $y$ and $x$ directions are the indentation and scratch directions, respectively. The substrate is a single crystal $(\mathrm{Au})$, which has a approximate size of $250 \times 200 \times 150 \AA^{3}$. The Embedded-Atom Method (EAM) potential for $\mathrm{Au}$ is taken from Ref. [13], simulations with other EAM potentials [14,15] are also compared in the study. Morse potential between the tip and the substrate is adopted according to Refs. [16,17], but with only repulsive interaction considered [7]. The Morse potential is written as:

$\phi\left(r_{i j}\right)=D\left\{\exp \left[-2 \alpha\left(r_{i j}-r_{0}\right)\right]-2 \exp \left[-\alpha\left(r_{i j}-r_{0}\right)\right]\right\}$

where $\phi\left(r_{i j}\right)$ is the pair potential energy function. $D, \alpha$, and $r_{0}$ correspond to the cohesion energy, the elastic modulus and the atomic distance at equilibrium, respectively. The potential parameters are set as $D=0.1 \mathrm{ev}, \alpha=1.70 \AA^{-1}, r_{0}=2.20 \AA$ for the interaction

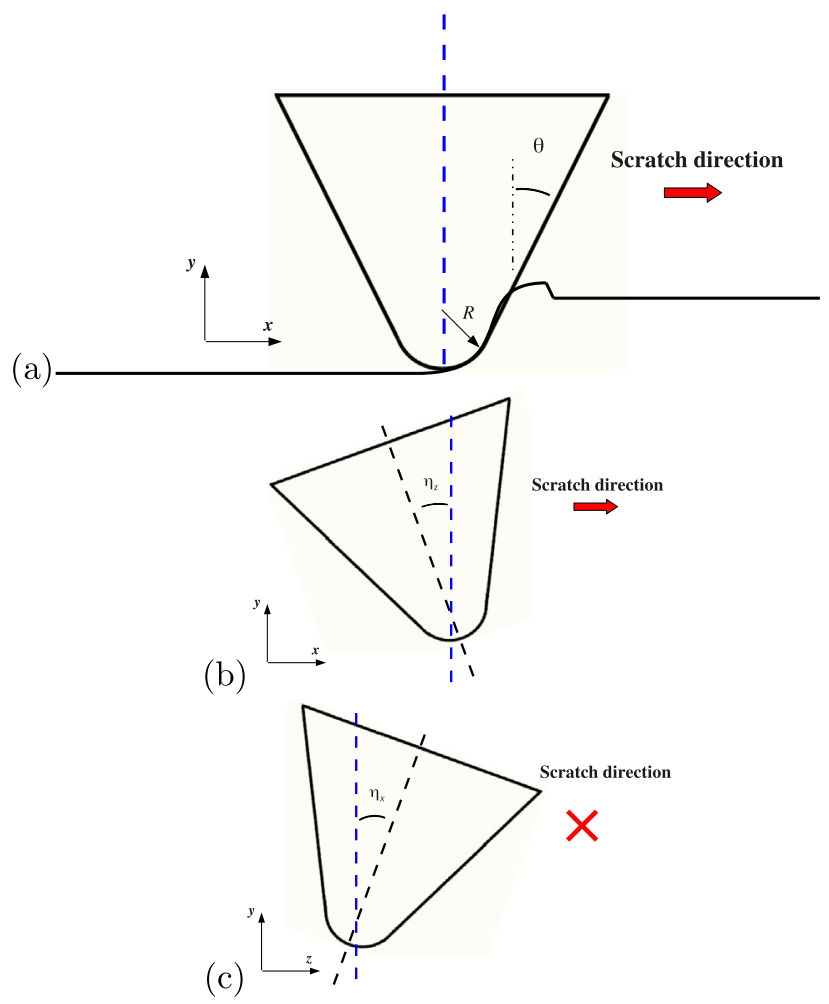

Fig. 1. Sketch of the simulation model (a) section view of $x-y$ plane (b) section view of $x-y$ plane with tilting backward $\left(\eta_{z}>0\right)$ (c) section view of $z-y$ plane with tilting laterally (changing $\eta_{x}$ ). The tilt angles shown here are in the positive direction. The cross is defined as the scratch direction is perpendicular to the paper.
Table 1

Crystal orientations of 11 simulation sets.

\begin{tabular}{|c|c|c|c|c|c|c|c|}
\hline Case ID & $\beta\left(^{\circ}\right)$ & & tations & & & & \\
\hline A & 0.00 & $x:$ & $\langle 00 \overline{1}\rangle$ & $y:$ & $\langle 110\rangle$ & $z:$ & $\langle 1 \overline{1} 0\rangle$ \\
\hline B & 8.05 & $x:$ & $\langle 1 \overline{1} \overline{10}\rangle$ & $y:$ & $\langle 110\rangle$ & $z:$ & $\langle 5 \overline{5} 1\rangle$ \\
\hline C & 10.03 & $x:$ & $\langle 1 \overline{1} \overline{8}\rangle$ & $y:$ & $\langle 110\rangle$ & $z:$ & $\langle 4 \overline{4} 1\rangle$ \\
\hline D & 13.26 & $x:$ & $\langle 1 \overline{1} \overline{6}\rangle$ & $y:$ & $\langle 110\rangle$ & $z:$ & $\langle 4 \overline{4} 1\rangle$ \\
\hline E & 19.47 & $x:$ & $\langle 1 \overline{1} \overline{4}\rangle$ & $y:$ & $\langle 110\rangle$ & $z:$ & $\langle 2 \overline{2} 1\rangle$ \\
\hline $\mathrm{F}$ & 35.26 & $x:$ & $\langle 1 \overline{1} \overline{2}\rangle$ & $y:$ & $\langle 110\rangle$ & $z:$ & $\langle 1 \overline{1} 1\rangle$ \\
\hline G & 54.74 & $x:$ & $\langle 1 \overline{1} \overline{1}\rangle$ & $y:$ & $\langle 110\rangle$ & $z:$ & $\langle 1 \overline{1} 2\rangle$ \\
\hline $\mathrm{H}$ & 64.76 & $x:$ & $\langle 3 \overline{3} \overline{2}\rangle$ & $y:$ & $\langle 110\rangle$ & $z:$ & $\langle 1 \overline{1} 1\rangle$ \\
\hline I & 74.21 & $x:$ & $\langle 5 \overline{5} \overline{2}\rangle$ & $y:$ & $\langle 110\rangle$ & $z:$ & $\langle 1 \overline{1} 5\rangle$ \\
\hline $\mathrm{J}$ & 79.98 & $x:$ & $\langle 4 \overline{4} \overline{1}\rangle$ & $y:$ & $\langle 110\rangle$ & $z:$ & $\langle 1 \overline{1} 8\rangle$ \\
\hline $\mathrm{K}$ & 90.00 & $x:$ & $\langle 1 \overline{1} 0\rangle$ & $y:$ & $\langle 110\rangle$ & $z:$ & $\langle 001\rangle$ \\
\hline
\end{tabular}

between the tip and substrate. The forces are recorded as the summation of interaction forces between the tip and the substrate.

Before the indentation process, each simulation process includes several steps: generation of samples from the perfect crystal lattice, energy minimization of sample by using conjugate gradient method, relaxing at the temperature of $600 \mathrm{~K}$ with pressure of 0 Pa under NPT ensemble (number of molecules $N$, pressure $P$ and temperature $T$ are conserved) for $50 \mathrm{ps,} \mathrm{system} \mathrm{cooling} \mathrm{down}$ to $1 \mathrm{~K}$ in $50 \mathrm{ps}$ under NPT, and the finally system being relaxed for another $50 \mathrm{ps}$ at the temperature of $1 \mathrm{~K}$ under NVT ensemble (number of molecules $N$, volume $V$ and temperature $T$ are conserved), The size of the box can be obtained by using NPT ensemble. Both indentation and nano-scratch are performed at the temperature of $1 \mathrm{~K}$ so that the thermal effect on micro-scale plasticity can be eliminated. The indentation is carried out at a penetration speed of $50 \mathrm{~m} / \mathrm{s}$. Before scratch, another 50 ps relaxation is applied (Longer relaxation time ( $100 \mathrm{ps)}$ ) is also carried out, it does not change the structure). From our verification tests, we find that the indentation rate does not influence the scratch process. The scratch velocity is taken as $15 \mathrm{~m} / \mathrm{s}$, which is relatively slow compared to most of the previous nano-scratch simulations $[7,9,10]$. The scratch depth is defined as the distance between the lowest atom of the tip and the height of the sample surface after scratch, the depth is taken as $24 \AA$ in all the later simulations.

The anisotropy of friction is an intrinsic property for all crystalline solids [18]. In the present work, we focus our major attention on studying the tip tilt effect on friction behavior, thus, in order to minimize crystal orientation effect on the friction, we choose eleven crystal orientations on (110) surface for attaining an average estimate. These orientations are shown in Table 1. Because of the two-fold symmetry of $(110)$ surface, the rotation angle $(\beta)$ of each set, with respect to the reference frame ${ }^{1}$, is evenly distributed from $0.00^{\circ}$ to $90.00^{\circ}$. The tip is tilted either around $x$ axis (lateral to scratch direction) or $z$ axis (along the scratch direction), with angle defined as $\eta_{x}$ and $\eta_{z}$, respectively. The signs of tilt angle $\left(\eta_{x}\right.$ and $\left.\eta_{z}\right)$ are defined according to right hand rule for angular quantities.

\section{Results and discussions}

\subsection{Ploughing friction response with tilt angle}

In most of the previous MD simulations, the friction curve has large fluctuations, it leads to the difficulty of quantitatively analyzing the results and comparing with experimental results. The physical origin of large fluctuation basically comes from the thermal dynamical characteristics of atomic interactions (high frequency vibration), thus it needs averaging process to minimize the effect

\footnotetext{
${ }^{1}$ Case $\mathrm{A}$ in Table 1 is selected as the reference frame
} 
from high frequency modes, e.g. phonon effects. In the present study, for Au single crystal (110) surface, nano-scratches over eleven orientations, as shown in Table 1, are averaged to eliminate the high frequency vibrations from nano-scale contact, and also to minimize the anisotropic effect. Typical friction curves are shown in Fig. 2 for cases with $\eta_{z}=-20^{\circ}$ and $\eta_{z}=20^{\circ}$, both results with averaging six and eleven cases are shown for comparison. It can be seen that although large deviation appears between different orientations, deviation between averaged values using six and eleven cases are small. Furthermore, convergence study is also carried out using the averaging scheme as show in Table 2. Typical friction responses with $\eta_{z}=20^{\circ}$ are shown in Fig. 3(a), it can be seen that the force variation between six and eleven averaging is small. The friction coefficient results using different averaging method (with three different tilt angles $0^{\circ},-20^{\circ}$, and $20^{\circ}$ around $z$ axis) are shown in Fig. 3(b). It should be noted that, in all the cases, samples are selected with their rotation angles $\beta$ nearly evenly distributed from $0.0^{\circ}$ to $90.0^{\circ}$. It can be seen that, with increased number of cases, the averaged friction coefficient is converged, nearly constant fluctuation can be obtained by using average of eleven cases. Thus, in the later simulations, all the results are averaged over eleven cases to minimize the anisotropic effect.

Potential sensitivity is checked in this study (see in Fig. 4), it shows the friction curves without any tilt $\left(\eta_{x}=\eta_{z}=0^{\circ}\right)$ by using three sets of EAM potentials [13-15]. It can be seen that the change of potential only has small effect on the friction response, the curves fluctuate but the average friction values are converged at the stable stage. It can be concluded the change of the potential does not affect the results in this study. In the following simulations, the EAM potential by Grochola et al. [13] is used. This potential was developed based on the first-principle calculations, and is suitable to simulate the local plastic behavior of gold during nanoscale contact [19].

Tip tilt effect on friction response is studied in this study. The variation of friction coefficient $\mu$ with the tilt angle is shown in Fig. 5, where the error bar denotes the standard deviation over the scratch distance from $50 \AA$ to $150 \AA$. Change of the tilt angle $\eta_{z}$ has a higher impact on $\mu$ than the change of $\eta_{x}$ does: Variation of $\eta_{z}$ from $0^{\circ}$ to $-20^{\circ}\left(20^{\circ}\right)$ affects the friction coefficient with a change of $-37.7 \%$ (23.2\%), while variation of $\eta_{x}$ from $0^{\circ}$ to $-20^{\circ}\left(20^{\circ}\right)$ only affects the friction coefficient with a change of $-7.2 \%(-7.1 \%)$. The tilt of the tip has a similar effect as the change of tip rake angle, which has been studied: Pei et al. [20] studied the tool rake angle effect on friction, their results showed that the friction coefficient $\mu$ increases approximately 2.2 times with the rake angle changing from $-45^{\circ}$ to $0^{\circ}$. Komanduri et al. [21] also studied the effect of tool rake angle on the exit failure in nano-metric cutting, they showed that the friction coefficient $\mu$ increases approximately 1.6 times with the rake angle changing from $-30^{\circ}$ to $0^{\circ}$. However, in these studies, the tool and the substrate either have the same thickness (2D cutting) [20], or are 2D materials [21], it is not proper for understanding 3D nano-scratch. In present work, it can be assumed that variation of $\eta_{z}$ from $-20^{\circ}$ to $20^{\circ}$ corresponds to change of the tool rake angle from $-40^{\circ}$ to $0^{\circ}$, and the $\mu$ increased 1.97 times from our study, which falls in the same range as in previous. However, the absolute value in our work is less than the value in previous studies [20,21], which may be due to the differences of inter-facial potentials, tool shape and dimensions. In the 3D cutting, the 'platic flow' of the material around the tip decreases the cutting force, which can not be considered in the $2 \mathrm{D}$ cutting system as in the studies [20,21]. The detailed study is discussed in the next section.

\subsection{Local plasticity analysis}

During scratch, the scratch force or drag force is along the scratch direction, so most of the time, the horizontal force makes the tip either tilting forward or backward to the scratch direction. In this section, we analyze the local plastic behavior beneath the tip while the tilt direction is forward or backward to the scratch direction.

The local deformation is measured by using continuum mechanics: the atomic local strain tensor $\epsilon$ is computed as a best fit to represent the local affine transformation. In the algorithm, firstly, a local deformation gradient $\boldsymbol{F}$ is calculated to best map the local deformation by:

$\boldsymbol{x}^{0} \rightarrow \boldsymbol{x}$

where $\boldsymbol{x}^{0}$ is a vector in the reference configuration, and $\boldsymbol{x}$ is a vector in the current configuration. The deformation at the atom $i$, which is $\boldsymbol{F}_{i}$, can be determined by:

Table 2

Cases used in averaging.

\begin{tabular}{ll}
\hline Number of cases & Case IDs \\
\hline 1 & A \\
2 & A, F \\
4 & A, D, G, J \\
6 & A, C, E, G, I, K \\
11 & A, B, C, D, E, F, G, H, I, J, K \\
\hline
\end{tabular}
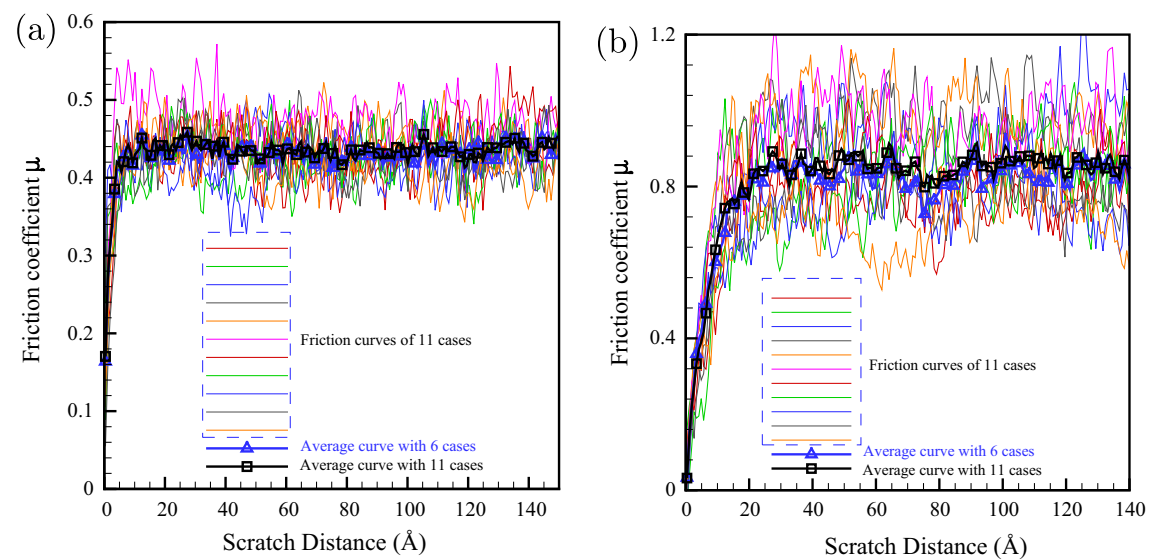

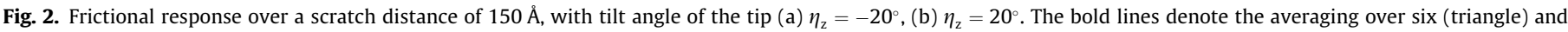
eleven (square) different crystal orientations. 

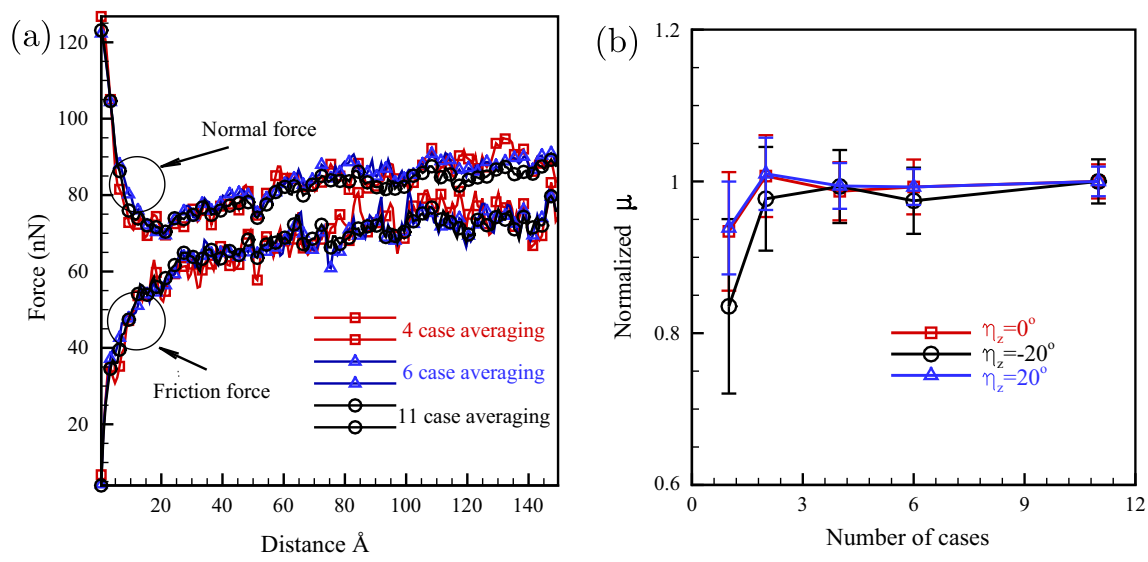

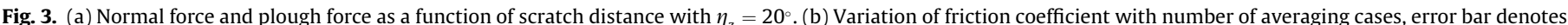

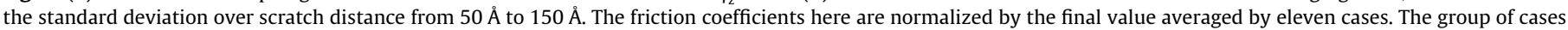
used in the averaging process are: one case-Case A; two cases-Case A and F; four cases-Case A,D, G and J; six cases-Case A,C,E,G,I and K.

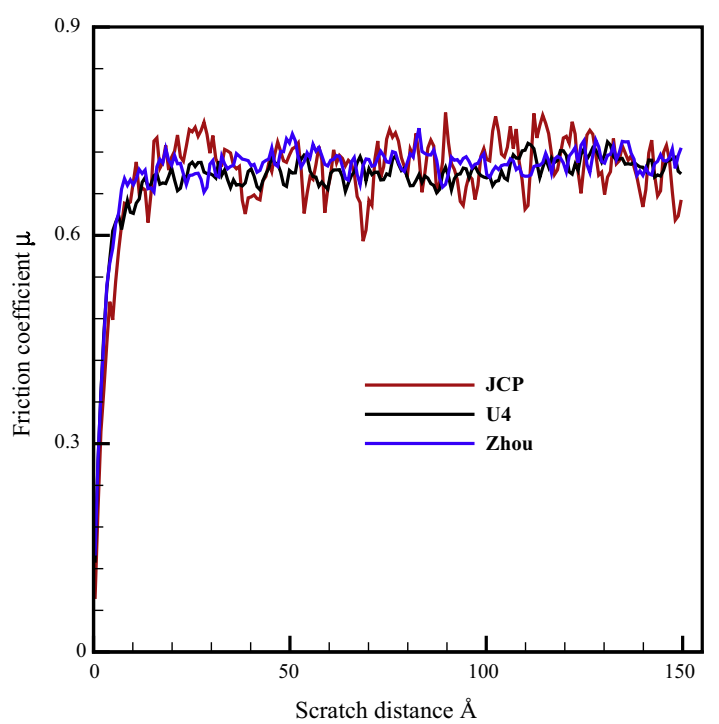

Fig. 4. Friction curves using different sets of potential with scratch depth $24 \AA$ and $\eta_{x}=\eta_{z}=0^{\circ}$, JCP denotes potential [13], U4 denotes potential [14], and Zhou denotes potential [15]. Each curve is averaged over eleven cases as in Table 1.

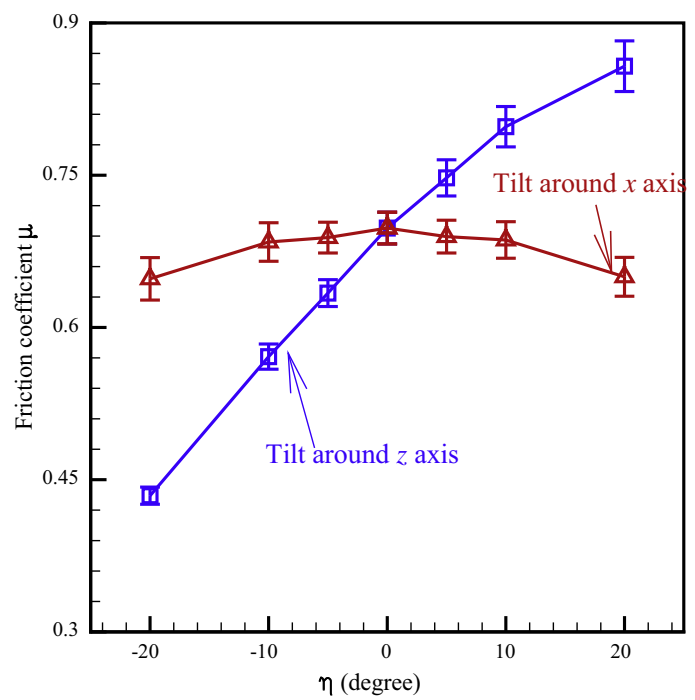

Fig. 5. Friction coefficient as a function of the tilt angle around $x$ or $z$ axis, error bar denotes the standard deviation over scratch distance from $50 \AA$ to $150 \AA$. $\min \left[\sum_{j=1 \rightarrow n}\left(\boldsymbol{F}_{i} \boldsymbol{x}_{i j}^{0}-\boldsymbol{x}_{i j}\right)^{2}\right]$

where $n$ is number of all the nearest atoms of atom $i$ in the initial configuration. then the local strain tensor at atom $i$ can be calculated by:

$\boldsymbol{\epsilon}_{i}=\frac{1}{2}\left[\boldsymbol{F}_{i} \boldsymbol{F}_{i}^{T}-\boldsymbol{I}\right]$

And finally, an equivalent strain $\epsilon_{v}$ is calculated to quantitatively measure the local deformation by:

$\epsilon_{v}=\sqrt{\epsilon_{12}^{2}+\epsilon_{23}^{2}+\epsilon_{13}^{2}+\frac{\left(\epsilon_{11}-\epsilon_{22}\right)^{2}+\left(\epsilon_{22}-\epsilon_{33}\right)^{2}+\left(\epsilon_{33}-\epsilon_{11}\right)^{2}}{6}}$

The computation procedure is detailed in the references $[22,23]$.

Fig. 6 shows the strain distribution under the tip (the cutting section view of $x-y$ ) at the scratch distance of $75 \AA$ for three different tilt angles $\eta_{z}$. It can be seen that the case with tip tilt direction [see in Fig. 6(a)], which is forward to the scratch direction, has the largest plastic area beneath the tip. The plastic area decreases, as the tip tilts to backward of the scratch direction with the increase of tilt angle [see in Fig. 6(c)]. The tilt effect on microstructure evolution is shown in Fig. 7, the atoms are colored according to local crystalline order analysis [24]. Large area of stacking fault and local slips (arrows in the figure) can be seen with $\eta_{z}=-20^{\circ}$, as shown in Fig. 7(a). Less stacking fault is found with increasing $\eta_{z}$ to $20^{\circ}$, but atomistic pile-up increases as $\eta_{z}$ increases (see in Fig. 7(b) and (c)).

The atomistic pile-up in front of the tip is critical for understanding the ploughing friction, an analytical solution has been proposed by Mishra et al. [7,8], but the tilt effect has not been studied. The effect of tilt angle on pile-up of atoms is shown in Fig. 8(a), the pile-up atoms accumulate linearly as the scratch processes, the pile-up volume with tilt angle $\eta_{z}=20^{\circ}$ is two times larger than the value with $\eta_{z}=-20^{\circ}$. The pile-up configurations after the scratch are shown in Fig. 9, where the orientation of substrate is chosen as case $\mathrm{A}$ in Table 1 . It demonstrates that the pile-up atoms increase as increase of the tilt angle, it is the ploughing who causes atomic accumulation of larger volume.

Quantitatively, the function of plastic area and the pile-up volume, at the scratch distance of $150 \AA$, to the tilt angle $\eta_{z}$ is shown in Fig. 10. As the tilt angle $\eta_{z}$ increases from $-20^{\circ}$ to $20^{\circ}$, the plastic area decreases more than $33 \%$; On the other hand, the pile-up volume increases more than twice. It can be concluded that, when the 
(a)

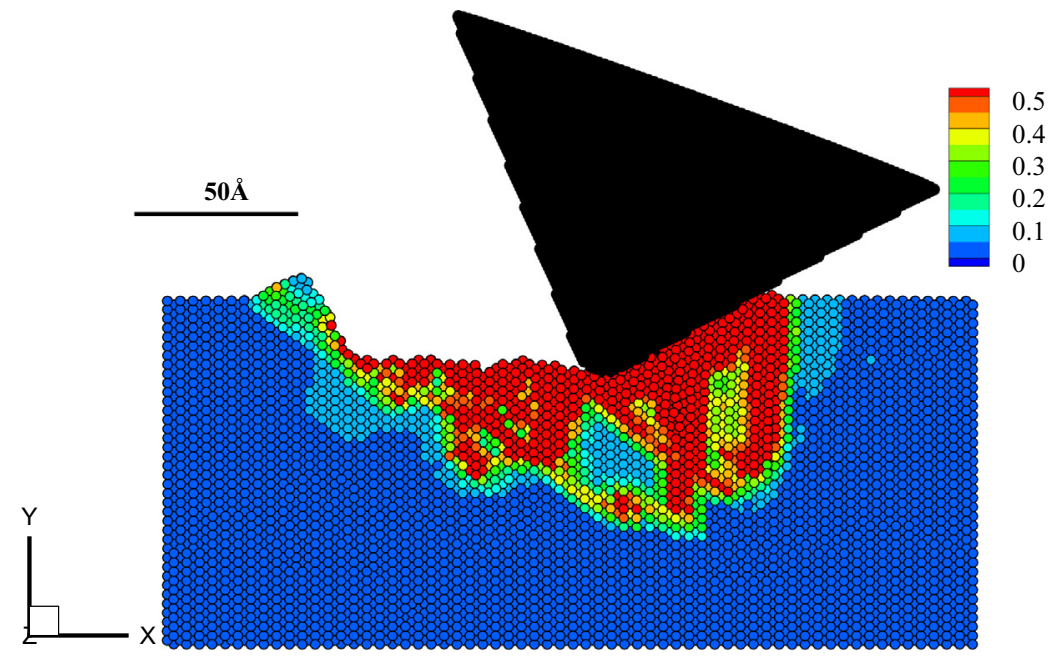

(b)
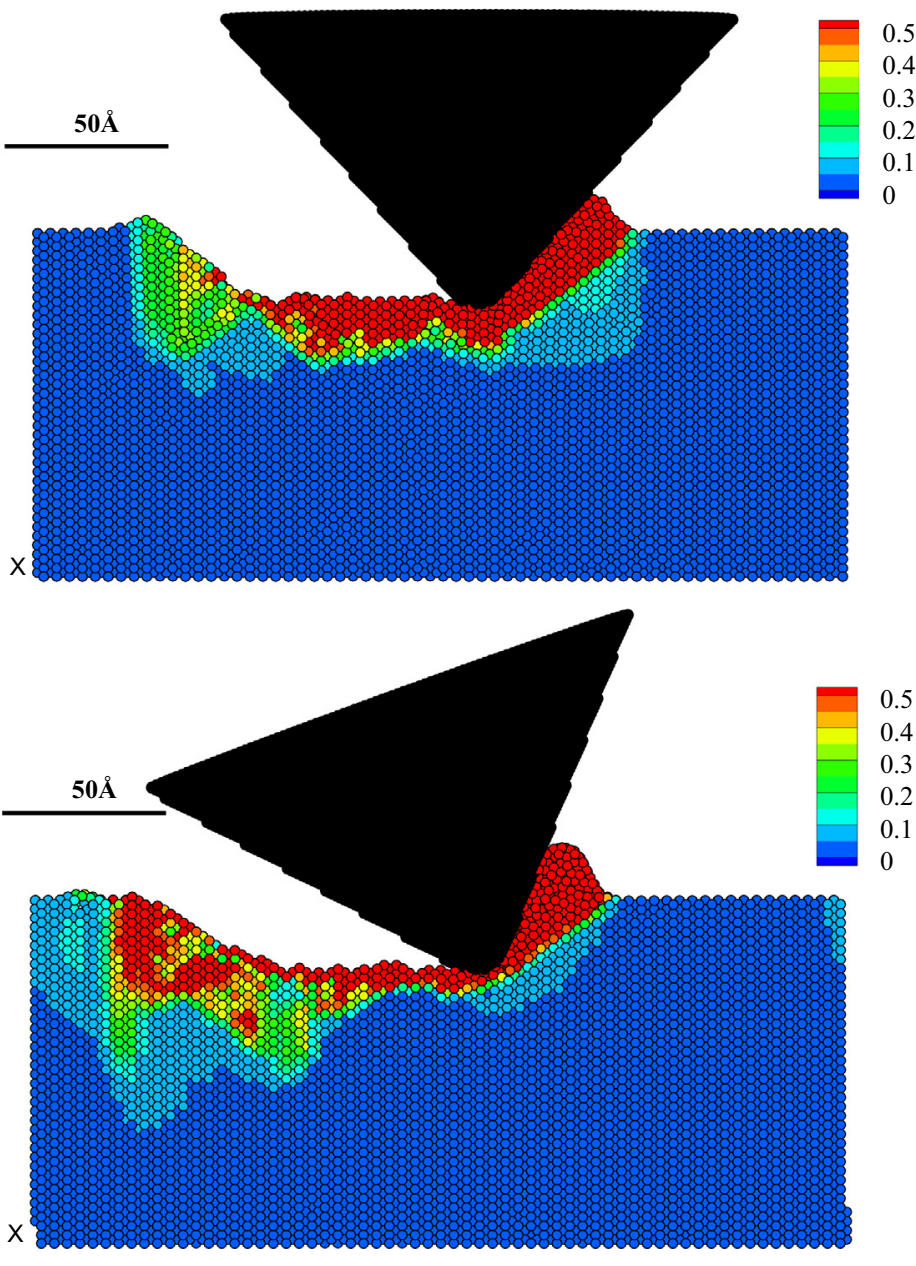

Fig. 6. $x-y$ section view of the strain $\epsilon_{v}$ distribution at the scratch distance of $75 \AA$ (a) $\eta_{z}=-20^{\circ}$ (b) $\eta_{z}=0^{\circ}$ (c) $\eta_{z}=20^{\circ}$.

tip tilting backward to the scratch direction, the nano-scale surface wear is severe because of the ploughing, but the sub-surface damage is less because of the smaller plastic regime beneath the tip.

\subsection{Nano-scale contact forces and hardness}

The effect of tilt on nano-hardness is studied in this section. The variation of the forces (normal force and cutting force) with $\eta$ is shown in Fig. 11, where the error bars denotes the standard deviation over the scratch distance of $50 \AA$ to $150 \AA$. The contact forces differ with different tilt directions:

- Tilt backward (forward) to the scratch direction: As shown in Fig. $11(\mathrm{a}), \eta_{z}$ changed from $-20^{\circ}$ to $20^{\circ}$, variation of the scratch force is less than $7 \%$, while normal force monotonically decrease with $52.1 \%$. It is pretty clear that the increase of coefficient in Fig. 5 is mainly attributed to the decrease of normal force. It is interesting to findthe pile-up of atoms doesn't affect the 
(a)

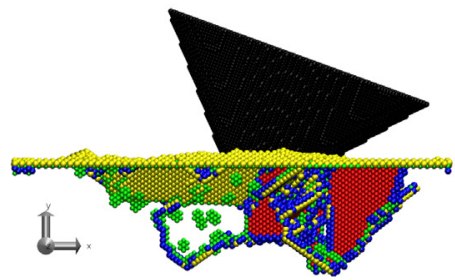

(c)

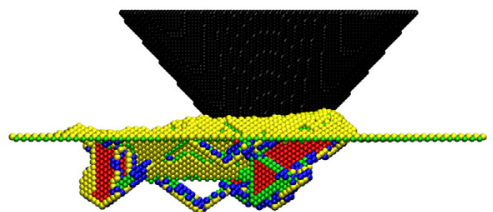

(d)

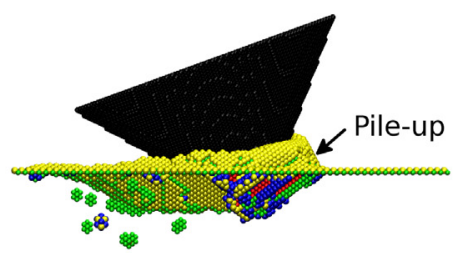

(b)
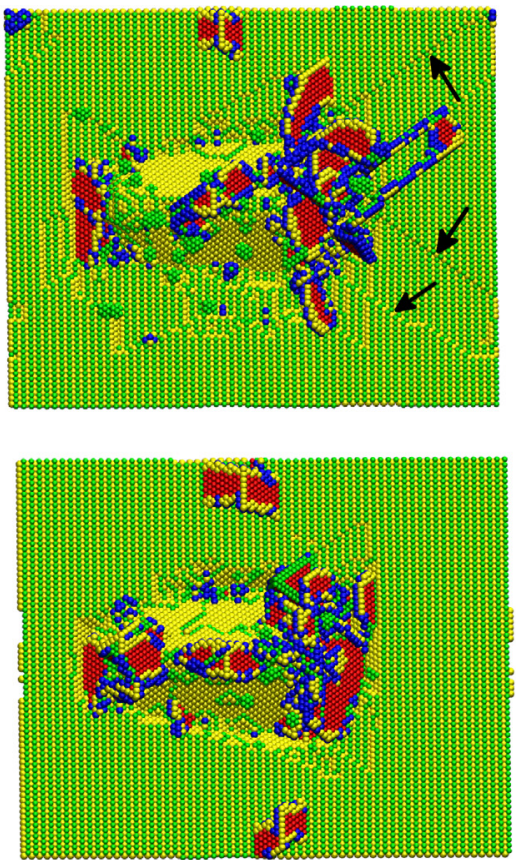

(f)

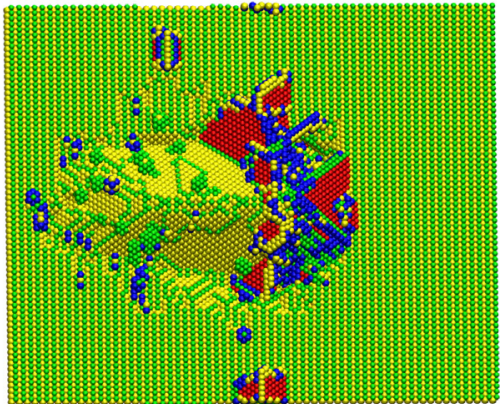

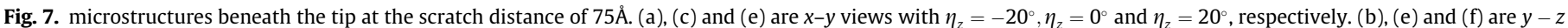

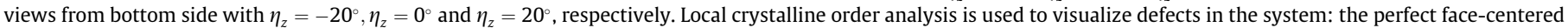

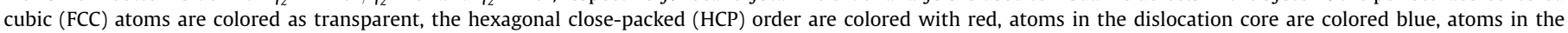

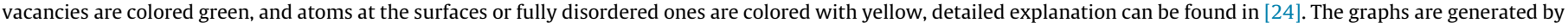
Visual Molecular Dynamics (VMD) [25]. (For interpretation of the references to color in this figure legend, the reader is referred to the web version of this article.)
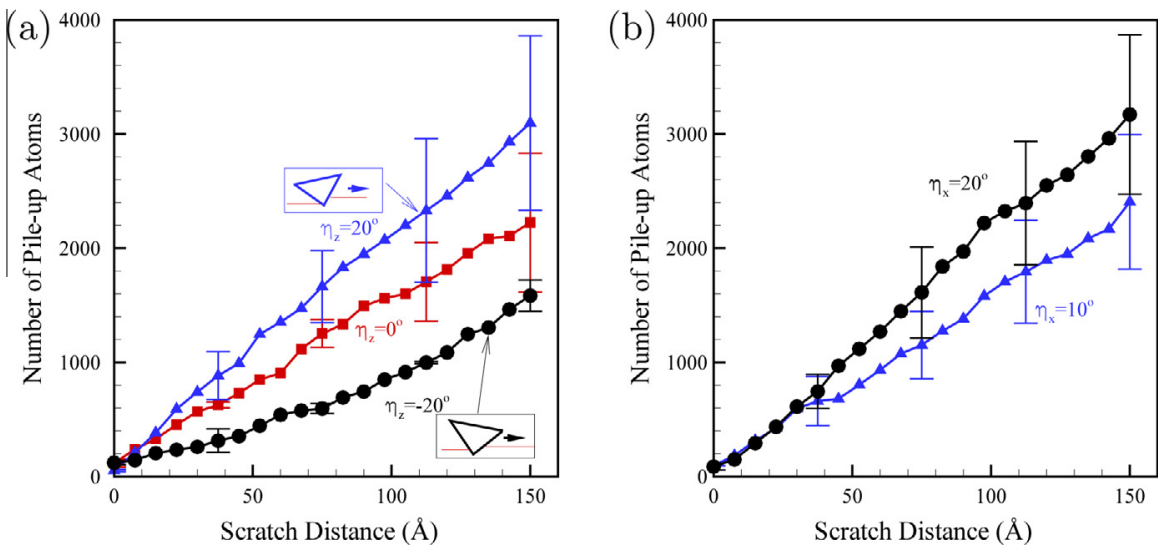

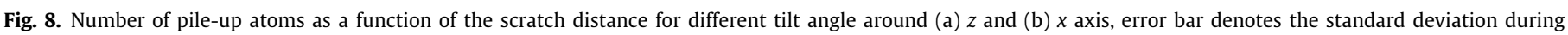
averaging over eleven cases.

scratch force very much, since the change of scratch force is approximately $7 \%$ but the volume change of pile-up atoms is approximately two times.
- Tilt laterally to the scratch direction: The tilt effect is larger on the normal force than the scratch force. As shown in Fig. 11(b), $\eta_{x}$ changes from $0^{\circ}$ to $-20^{\circ}\left(20^{\circ}\right)$, change of the scratch force 


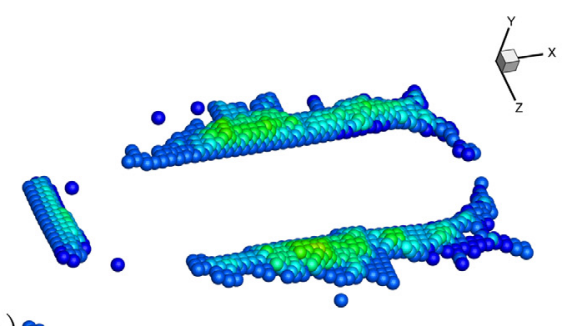

(a) $\infty$

(b)
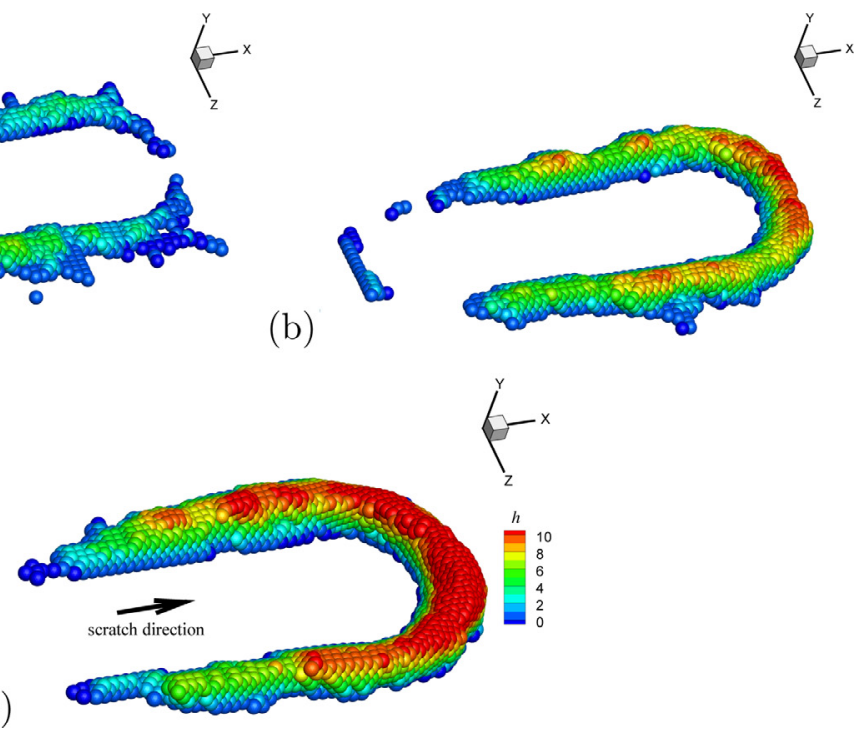

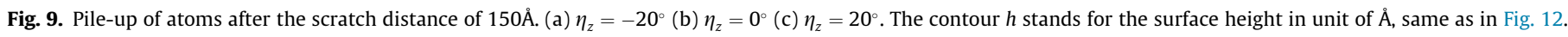

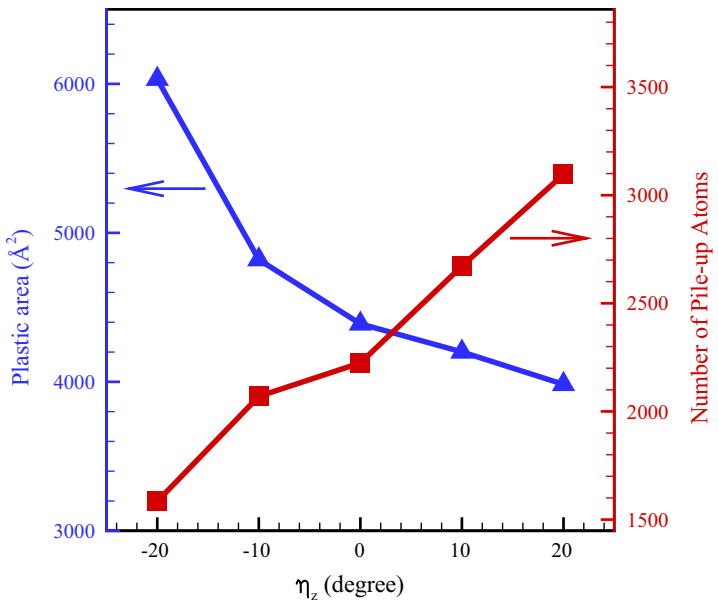

Fig. 10. Plastic area, number of pile-up atoms as a function of tilt angle $\eta_{z}$. increases $10.0 \%$ (10.1\%), while normal force increases $20.1 \%$ (18.9\%). The change of forces can also be reflected by the change of pile-up volume [see in Figs. 8(b) and 12], the volume of pile-up for $\eta_{x}=20^{\circ}$ is larger than $\eta_{x}=10^{\circ}$.

It can be concluded that normal force is more sensitive to the tip tilt rather than scratch force, it is also interesting to see that the pile-up of atoms does not play a major role on the change of scratch force. However, the pile-up of atoms affect the normal force, it seems that tilt of the tip should be carefully considered while measuring the normal hardness during scratch.

As discussed by Mo et al. [1] and Musser [2], the definition of atomic volume and area is not easy, especially for atoms within nano-scale yielding. So, it is absurd to define the yield stress or hardness in the atomic level. In this study, the contact atoms are defined as the gold atoms whose distance to the tip (all the carbon atoms) is less than $r_{0}$, where $r_{0}$ is the equilibrium distance defined in the Morse potential, as in Eq. (1). We calculate the contact force per atom as a quantity to measure the yielding during
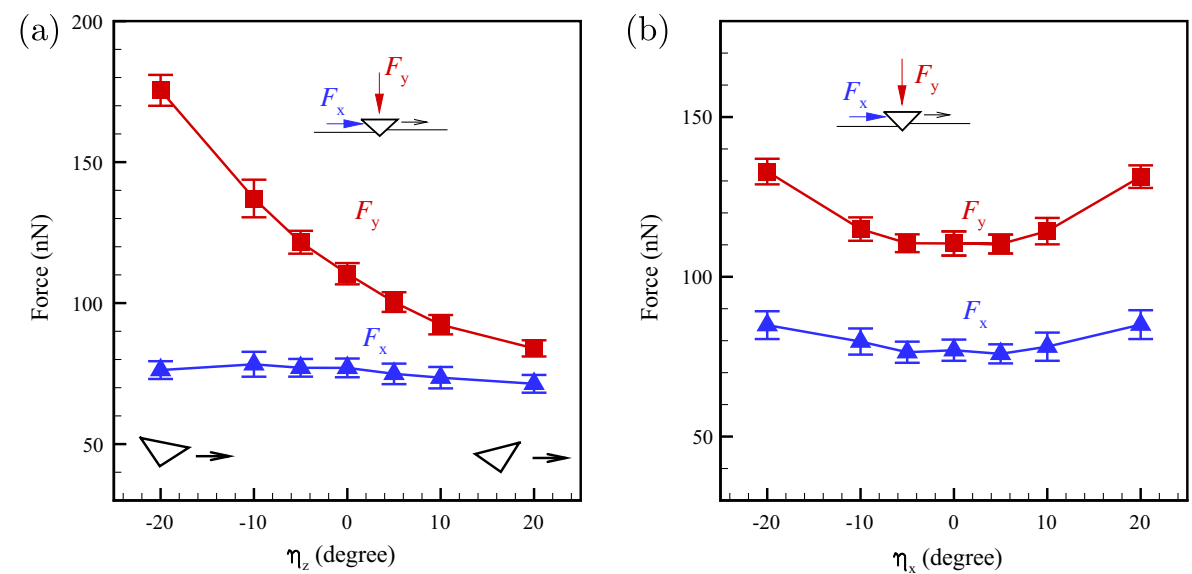

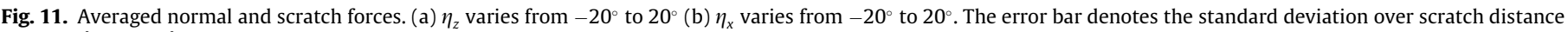
from $50 \AA$ to $150 \AA$ 
(a)
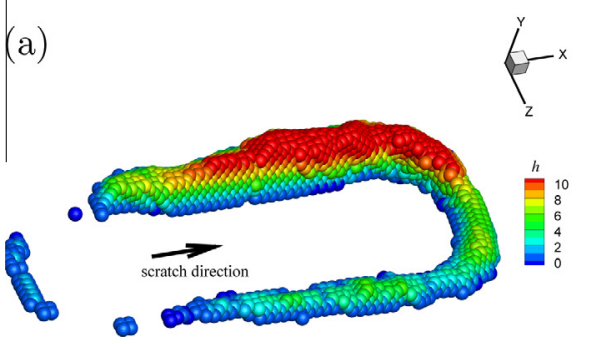

(b)

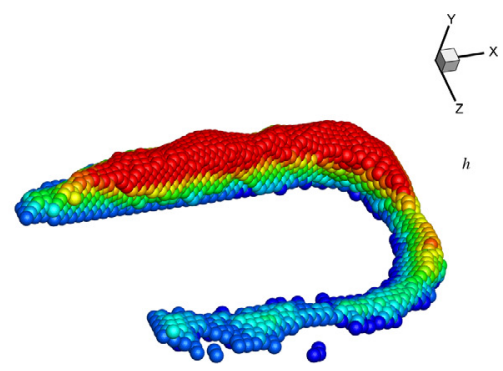

Fig. 12. Pile up of atoms after the scratch distance of $150 \AA$. (a) $\eta_{x}=10^{\circ}$ (b) $\eta_{x}=20^{\circ}$.

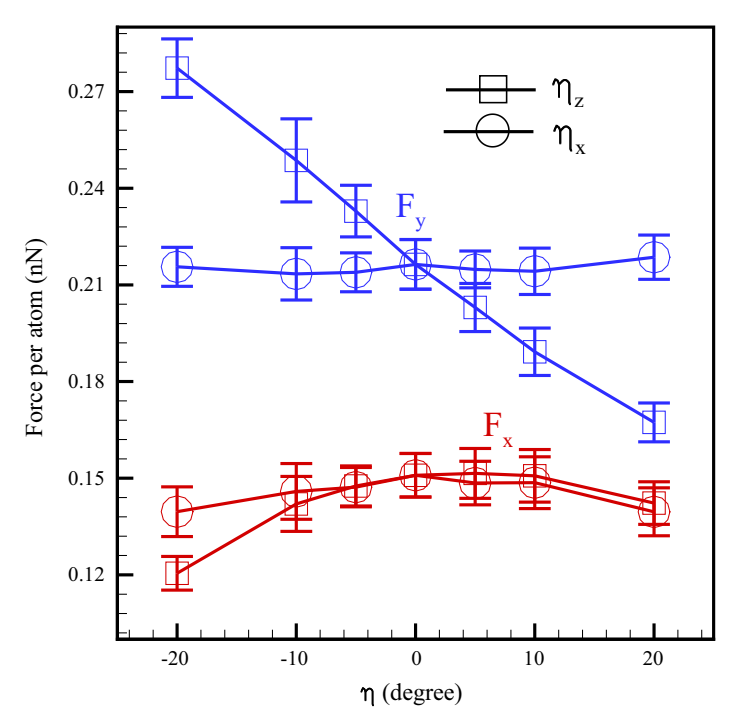

Fig. 13. Forces applied per atom during scratch as a function of tilt angle $\eta$.

nano-scratch. As shown in Fig. 13, when the tilt angle $\eta_{z}$ increases from $\eta_{z}=-20^{\circ}$ to $\eta_{z}=20^{\circ}$, the scratch force $f_{x}$ per atom varies approximately $19.0 \%$, while the normal force $f_{y}$ per atom decreases linearly 39.5\%; When the tilt angle $\eta_{x}$ increases from $\eta_{x}=-20^{\circ}$ to $\eta_{x}=20^{\circ}$, the scratch force $f_{x}$ per atom varies approximately $6.7 \%$, while the normal force $f_{y}$ per atom varies less than $2.0 \%$. It can be concluded that the tilt effect on hardness (normal force per atom) is larger when tilting in $\eta_{z}$ direction than in $\eta_{x}$ direction, the tilt effect of hardness in $\eta_{x}$ direction can be neglected (less than $2.0 \%)$. It can be concluded that measurement of the cutting hardness is much more accurate than the normal hardness in the scratch test, tilt of the tip should be strictly controlled if the normal hardness is the value for consideration.

\section{Conclusion}

Tip tilt effect on nano-scratch is studied by using MD simulation in the present work, conclusions are as follows:

- Tilting forward (backward) of the tip to the scratch direction has a larger effect on the friction coefficient than tilting laterally, the tilt effect on the normal force is the main reason for the change of friction coefficient. It is found that the tilt effect on the scratch force is much less than the effect on the normal force.

- Surface wear (pile-up of atoms) is the main mechanism when the tip tilts backward to the scratch direction. On the other side, subsurface plastic damage(dislocation plasticity) dominated when the tip tilts forward to the scratch direction.
- The normal hardness is sensitive to the tip tilting forward (backward), while the effect can be neglected if tip tilts laterally.

\section{Acknowledgements}

We acknowledge the financial support from the National Natural Science Foundation of China (Nos. 11202214, 91216108, 11302115), the National Basic Research Programs of China (No. 2012CB937500), from the State Key Laboratory of Mechanics and Control of Mechanical Structures (NUAA No. MCMS-0114G01), and the CAS/SAFEA International Partnership Program for Creative Research Teams. We thank the insightful discussions with Prof. Erik Van der Giessen from Groningen University, the Netherlands. We thank the useful comments from Prof. Zhuping Huang from Peiking University, China.

\section{References}

[1] Y. Mo, K.T. Turner, I. Szlufarska, Nature 457 (7233) (2009) 1116-1119.

[2] A. Musser, Friction on the nanoscale and the breakdown of continuum mechanics, <http://www.rug.nl/research/zernike/education/topmasternanoscience/ ns190musser.pdf>, May 2009.

[3] V.N. Koinkar, B. Bhushan, J. Mater. Res. 12 (12) (1997) 3219-3224.

[4] Z.-H. Xu, X. Li, Phil. Mag. 87 (16) (2007) 2299-2312.

[5] M.S. Kashani, V. Madhavan, Acta Mater. 59 (3) (2011) 883-895.

[6] C. Shi, H. Zhao, H. Huang, S. Wan, Z. Ma, C. Geng, L. Ren, Tribol. Int. 60 (2013) 64-69.

[7] M. Mishra, I. Szlufarska, Tribol. Lett. 45 (3) (2012) 417-426.

[8] M. Mishra, P. Egberts, R. Bennewitz, I. Szlufarska, Phys. Rev. B 86 (4) (2012) 045452-045460.

[9] Y. Gao, C. Lu, N. Huynh, G. Michal, H. Zhu, A. Tieu, Wear 267 (11) (2009) 19982002.

[10] P.-z. Zhu, Y.-z. Hu, H. Wang, T.-b. Ma, Mater. Sci. Eng: A 528 (13) (2011) 45224527.

[11] J. Kiely, J. Houston, Phys. Rev. B 57 (19) (1998) 12588-12594.

[12] S. Plimpton, J. Comput. Phys. 117 (1) (1995) 1-19.

[13] G. Grochola, S.P. Russo, I.K. Snook, J. Chem. Phys. 123 (2005) 204719-1204719-7.

[14] J. Adams, S. Foiles, W. Wolfer, J. Mater. Res. 4 (01) (1989) 102-112.

[15] X. Zhou, R. Johnson, H. Wadley, Phys. Rev. B 69 (14) (2004) 144113-1-14411310.

[16] T. Kitagawa, A. Kubo, K. Maekawa, Wear 202 (2) (1997) 142-148.

[17] T.-H. Fang, C.-I. Weng, Nanotechnology 11 (3) (2000) 148-153.

[18] R. Komanduri, N. Chandrasekaran, L. Raff, Wear 240 (1) (2000) 113-143.

[19] J. Wang, F. Sansoz, J. Huang, Y. Liu, S. Sun, Z. Zhang, S.X. Mao, Nat. Commun. 4 (2013) 1742-1748.

[20] Q. Pei, C. Lu, F. Fang, H. Wu, Comput. Mater. Sci. 37 (4) (2006) 434-441.

[21] R. Komanduri, N. Chandrasekaran, L. Raff, Mater. Sci. Eng.: A 311 (1) (2001) 112.

[22] X. Liu, F. Yuan, Y. Wei, Appl. Surf. Sci. 279 (2013) 159-166.

[23] F. Shimizu, S. Ogata, J. Li, Mater. Trans. 48 (11) (2007) 2923-2927.

[24] X. Liu, Z. Liu, Y. Wei, Tribol. Lett. 46 (2) (2012) 167-178.

[25] W. Humphrey, A. Dalke, K. Schulten, Vmd: visual molecular dynamics, J. Mol. Graph. 14 (1) (1996) 33-38. 